\title{
Acute Arthroscopic Removal of a Bullet Embedded in the Glenoid: A Case Report
}

\author{
Jonathan Guevara ${ }^{1}$, Karthikeyan Chinnakkannu', Sivashanmugam Raju ${ }^{1}$ \\ Learning Point of the Article: \\ Shoulder arthroscopy can successfully retrieve an even larger embedded bullet. Posterosuperior glenoid defects do not compromise \\ shoulder stability.
}

\section{Abstract}

Introduction: Retained bullets in joint spaces have been shown to cause both mechanical and chemical damage to the joint surfaces, leading to the risk of arthritis if untreated.

Case Report: A case of arthroscopic treatment for a gunshot to the shoulder with a retained bullet embedded in the glenoid is presented. The patient presented with multiple gunshots to his back and extremities, including gunshot to the left leg with popliteal vein injury, gunshot to the back with lumbar level laminar fractures and acute spinal cord injury, and gunshot to the left shoulder with a retained bullet in the posterior superiorglenoid.

Conclusion: Shoulder arthroscopy can be successfully employed to retrieve larger embedded bullet or foreign body with the help of tools such as laparoscopic graspers. Even with significant glenoid bone defect, the stability of the shoulder joint does not get compromised if the bone defects are located posterosuperiorly.

Keywords: Retained bullet, shoulder arthroscopy, gunshot, glenoid bone defect, humeral head fracture.

\section{Introduction}

Gunshot wounds with retained bullets in joint spaces represent a unique challenge to the orthopedic surgeon; potential risk for infection, lead intoxication, ongoing damage to chondral surfaces, and the acidic synovial environment all drastically increase the risk of arthropathy if unaddressed $[1,2]$.

Due to the ease of access to the glenohumeral joint and optimal visualization with minimal exposure and less soft-tissue disruption, we present a case of arthroscopic shoulder irrigation and debridement, including arthroscopic bullet removal as the preferred method for assessing the shoulder after gunshot injuries.

\section{Case Report}

A 17-year-old male presented to the pediatric trauma hospital emergency department after an altercation with multiple gunshot wounds. As per advanced trauma life support protocol, life-threatening injuries were ruled out systematically. Orthopedic service was consulted to assess his injuries, which included three gunshot wounds of particular orthopedic interest. He sustained a gunshot wound to the medial aspect of the left thigh with profuse popliteal bleeding, gunshot to the right flank with missile air tracks down to the level of the posterior elements of the lumbar spine with resultant right L2 pedicle and laminar fractures, and finally, a left shoulder gunshot wound just inferior to the acromial margin in a lateral to the medial trajectory. Imaging revealed retained bullet fragments lodged in the posterosuperior quadrant of the glenoid (Fig. 1,2).

At initial presentation, while he was hypotensive and tachycardic, physical examination was notable for no sensation

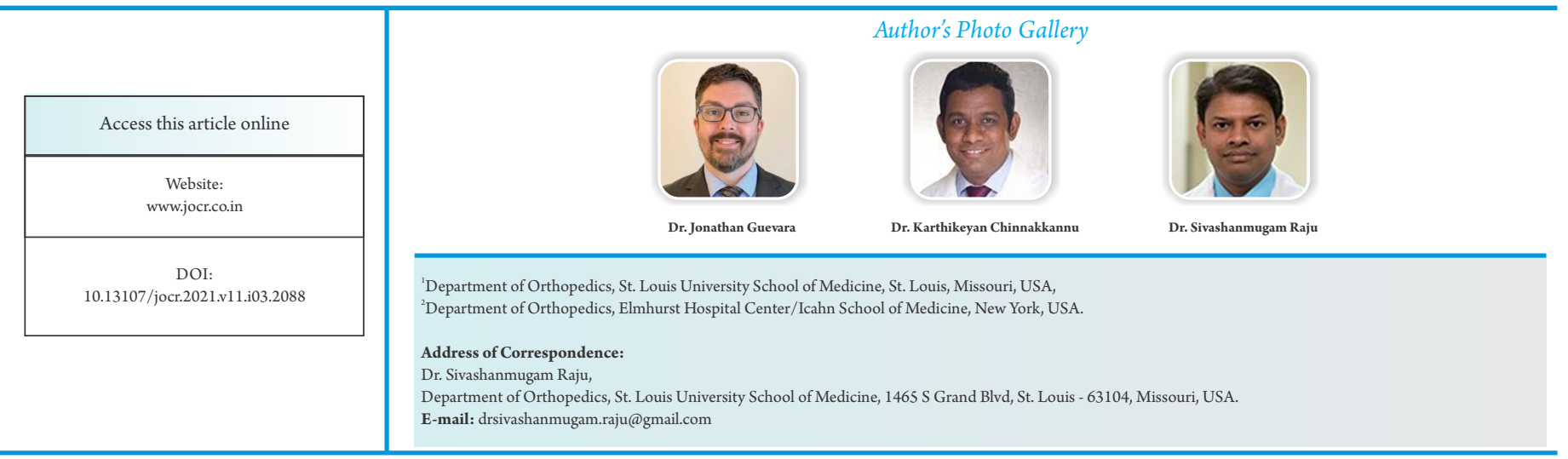

Journal of Orthopaedic Case Reports | pISSN 2250-0685 | eISSN 2321-3817 | Available on www.jocr.co.in | doi:10.13107/jocr.2021.v11.i03.2088 This is an Open Access article distributed under the terms of the Creative Commons Attribution Non-Commercial License (http://creativecommons.org/licenses/by-nc/3.0) which permits unrestricted non-commercial use, distribution, and reproduction in any medium, provided the original work is properly cited. 


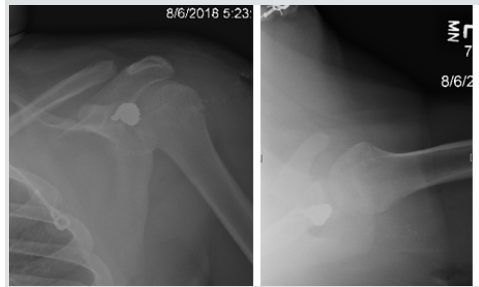

Figure 1: Radiographic image (anterior-posterior, lateral, and Scapular Y view) showing bullet in the glenoid.
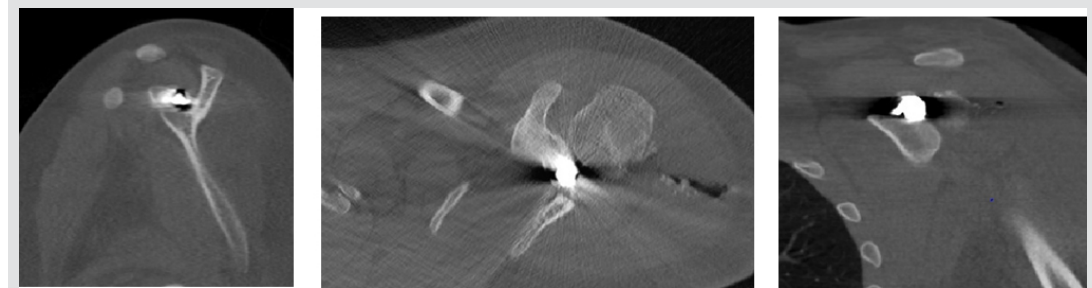

Figure 2: Computed tomography (axial and coronal) images showing the impacted bullet in the posterosuperior quadrant of glenoid.

or motor control below the level of the umbilicus. Emergently, he was taken to the operating room for exploratory laparotomy as well as the vascular exploration of the left popliteal injury. Exploratory laparotomy revealed superior pole renal and caudate lobe hepatic lacerations which were hemostatic. The popliteal fossa was explored once proximal control was gained at the level of the groin and revealed considerable popliteal vein injury without arterial injury. Once hemostasis was obtained, fasciotomies were performed for the lower leg due to the significant time under tourniquet control before surgery. Osseous injuries to the spinal column were deemed stable, suitable for upright positioning, and the plan was to treat them conservatively.

Due to the multiple injuries and critical status, his early care was dominated by intensive resuscitation, and once he was deemed adequately resuscitated for additional surgery, it was planned to address the retained bullet and shoulder joint arthroscopically. Of particular interest in addressing the left shoulder was the diagnosis of acute paraplegia with possible incomplete spinal cord injury, which would necessitate as much preserved upper extremity function as possible. The decision was made in concert with the surgical and intensive teams to continue intravenous antibiotic coverage until it was deemed safe to address the shoulder surgically. The pre-operative computed tomography (CT) of the shoulder (Fig. 2) was carefully studied, and the bullet was located in the posterosuperior quadrant of the glenoid. The bullet measured $1.6 \mathrm{~cm}$ in diameter which is large and the normal arthroscopic graspers would not be adequate. Hence, various other instrument options were explored, including laparoscopic graspers.

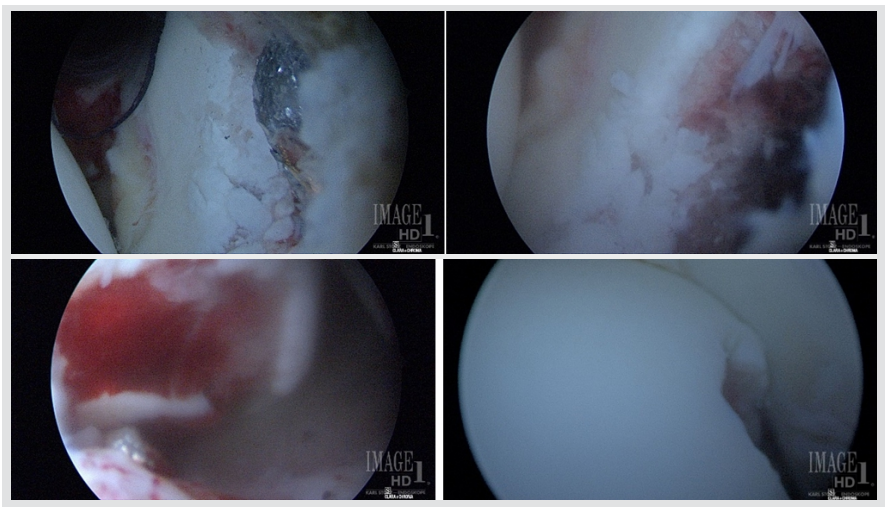

Figure 3: Arthroscopic image showing the bullet, glenoid after bullet removal, humeral head defect, and relation of head and glenoid articulation.

\section{Procedure}

On hospital day 4, the patient was brought to the operating room. He was positioned on a regular table with beach chair extension, seated upright at $70^{\circ}$ with the head and chest secured to the head positioner and the table. The left arm was prepped and draped free in standard fashion, with the pectoral girdle freely mobile. The forearm was secured in a pneumatic releasestatic arm holder attached to the bed. A standard posterior viewing portal in the joint was made, and the joint was inspected. Diagnostic arthroscopy revealed the following (Fig. $3)$ : There was rent in rotator cuff posteriorly, and a large defect was noted along with the posterior humeral head and bare area resembling a large atypical Hill-Sachs lesion. The superior labrum was frayed and partially absent at the posterior superior margin, and the glenoid at the posterior superior quadrant had $30-40 \%$ surface area impaction with obvious retained metallic bullet and jacket fragments. Otherwise, the cartilage and bony glenoid appeared healthy (Fig. 3). There were small osteochondral fragments partially attached to the periphery of the humeral head defect and scattered small metallic pieces.

The posterosuperior labral fraying was debrided with a shaver and cautery wand. All loose fragments and partially attached osteochondral fragments were removed with the shaver or a grasper. Satisfied with the progress at that point, attention was turned to the retained bullet. An accessory mid-lateral portal was created to have better direct access to manipulate (straight shot) the bullet using a freer from within the glenoid vault. Once this was freed to the point of mobility, the anterior working

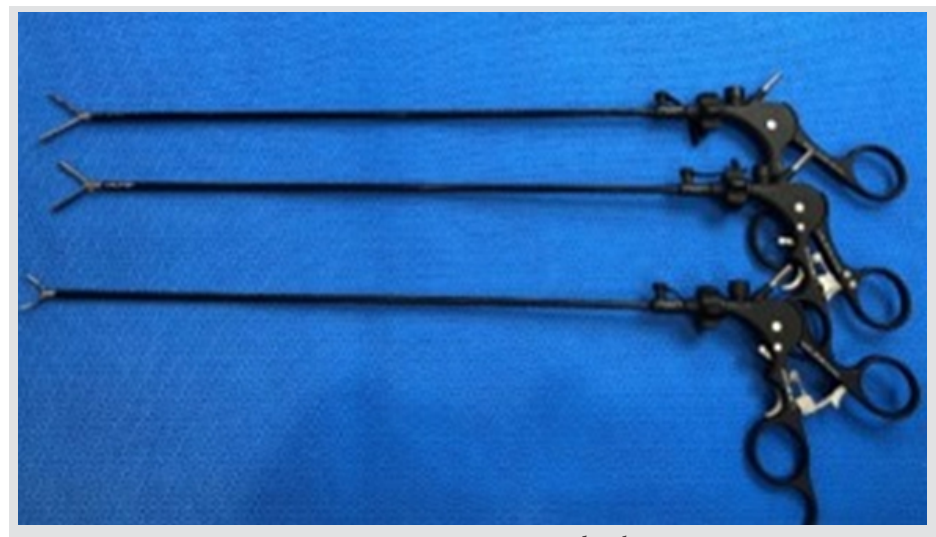

Figure 4: Laparascopic graspers used in this case. 

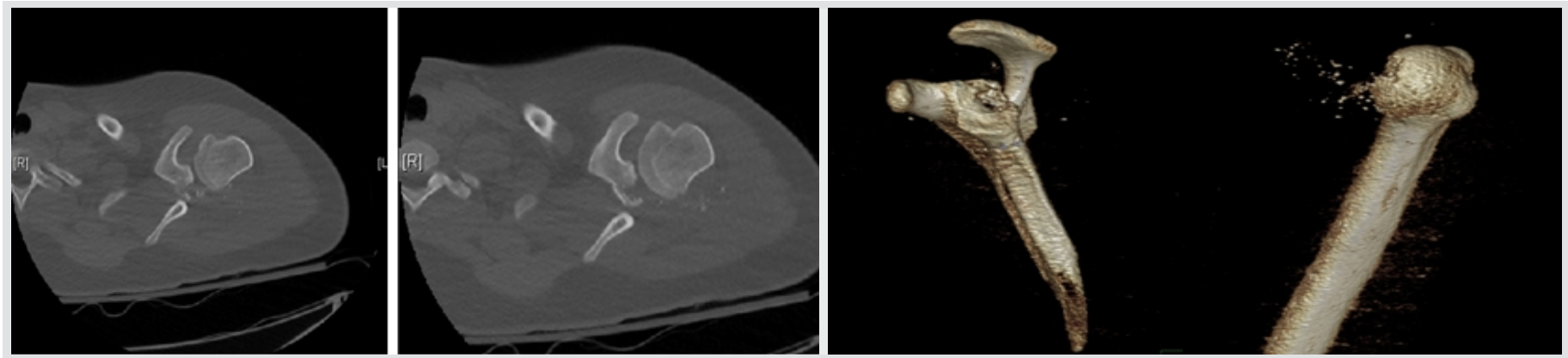

Figure 5: Post-operative computed tomography, including 3D reconstruction showing the glenoid and humeral head defect.

portal was enlarged (12 mm cannula), and a standard grasper was used to attempt seizing the piece in its entirety. This was unsuccessful due to the sheer size of the retained bullet. A laparoscopic instrument set (Fig. 4) was opened, and the long graspers were used to pull the piece from the lateral portal and seize it within the joint. The laparoscopic graspers did wide open and were also able to lock after grasping the bullet. This allowed us to use force to pull the bullet from the glenoid. The bullet was then secured by an additional pair of laparoscopic locking graspers through the anterior working portal, and the bullet was delivered completely from this portal along with the cannula, as the bullet was larger than the cannula, in a twisting motion as one piece. Following this, the camera remained in the shoulder joint through the posterior viewing portal, and the shoulder was taken through a range of motion to inspect for any engagement of the humeral head and glenoid defects. The humeral head defect did not engage with the glenoid in abduction and external rotation. The glenoid defect occupied the posterior portion of the superior glenoid, and there was a posterior glenoid rim and labrum intact, so there was not a concern for instability at that time. Post-operative CT scan of the shoulder showed 30\% articular bone loss (Fig. 5); however, clinically, the shoulder was stable.

At a 1.5 year follow-up, the patient denies any pain in the shoulder. His paraplegia was recovering, and his strength had improved to 3/5 at knee and hip on both sides. Regarding the left upper extremity, examination revealed a painless active range of motion and well-healed surgical incisions from the arthroscopy. The patient has had symmetrical forward flexion, abduction, and internal and external rotations. The rotator cuff strength was normal except for slight weakness of external rotation compared to the opposite side. There was no anterior apprehension. The recent follow-up radiographs shoulder joint space was maintained (Fig. 6). Informed consent has been obtained from the patient who is 18 years old now.

\section{Discussion}

Arthroscopic removal of bullets from the shoulder has been described $[3,4,5,6]$ and they are technically challenging.
Those reports include removal of bullet from subacromial space $[3,6]$, posterior recess [3], or supraspinatus fossa [5]. Entry wounds should be debrided of all foreign and surface material, including clothing, skin, and hair. Retained lead-containing bullets can function as devastating foreign bodies, while the acidic environment may elute lead from the bullet which can lead to lead arthropathy [7], hypertrophic, or fibrotic changes [3]. Bullets embedded in the articular surface can cause mechanical damage to the articular cartilage during joint movements [8]. The arthroscopic removal offers the advantage of direct visualization with minimal soft-tissue disruption. In the case where direct visualization of the superior and posterior glenoid surface is desired, the arthroscopic approach gives a distinct advantage over the open approach in the case of a native joint making posterior approaches, excessive humeral retraction, and glenoid releases unnecessary adjuncts to exposure. Assessing bone loss may be achieved by postoperative CT scan, which was performed here; however, an inspection of the quality of bone, any fracture extension, and the stability of the posterior glenoid rim is best assessed by arthroscopic visualization and probing. In general, posterior instability is uncommon, and bone block procedures indicated only in symptomatic patients glenoid bone loss of $>20 \%$ with instability [9] or bone loss of more than $10 \%$ with engaging humeral head defects [10]. In our patient, the bone loss was in the posterior-superior quadrant, and it was more than $30 \%$. However, during our examination, it did not contribute or cause any instability, possibly due to his intact posterior glenoid rim and labral buttress seen at the time of initial arthroscopy. Moreover, his humeral head defect was non-engaging, and also, the mechanism of injury may also contribute to the stability, as he did not suffer a dislocation event, only a penetrating trauma

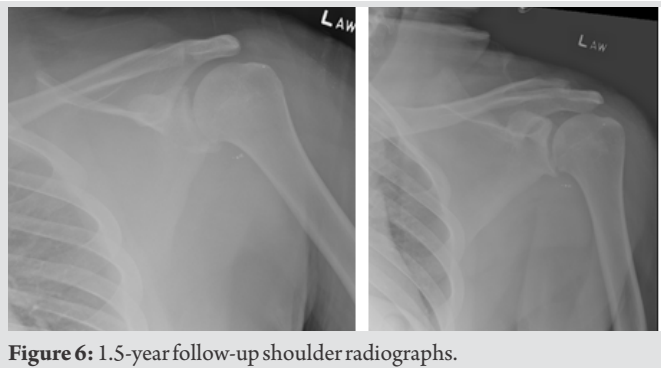


which only confers soft-tissue disruption along the missile track, sparing the constellation of soft-tissue injuries seen in the blunt dislocation events.

Our case is unique as the bullet was large and as well as embedded in the bone as opposed to free-floating bullets in the previous studies $[3,4,5,6]$, which could be considered loose bodies. In our case, the bullet measured $1.6 \mathrm{~cm}$ (diameter), and it was not possible to grasp with regular arthroscopic graspers. The depth of the joint, especially in this case, precludes the use of non-arthroscopic grasping tools such as a Kocher or Ochsner clamp, as these will not open sufficiently through a narrow tunnel. In these situations, laparoscopic locking graspers are very helpful. They are larger and can safely lock while grasping the foreign body.

\section{Conclusion}

Even with the larger embedded bullet or foreign body, we recommend utilizing an arthroscopic approach for irrigation and debridement after a gunshot wound to the shoulder with

\section{References}

1. Rehman MA, Umer M, Sepah YJ, Wajid MA. Bullet-induced synovitis as a cause of secondary osteoarthritis of the hip joint: A case report and review of literature.J Med Case Rep 2007;1:171.

2. Windler EC, Smith RB, Bryan WJ, Woods GW. Lead intoxication and traumatic arthritis of the hip secondary to retained bullet fragments. A case report. J Bone Joint Surg Am 1978;60:254-5.

3. Mahirogullari M, Cilli F, Akmaz I, Pehlivan O, Kiral A. Acute arthroscopic removal of a bullet from the shoulder. Arthroscopy 2007;23:676.

4. Tarkin IS, Hatzidakis A, Hoxie SC, Giangara CE, Knight RQ. Arthroscopic treatment of gunshot wounds to the shoulder. Arthroscopy 2003;19:85-9.

5. Galland A, Lunebourg A, Airaudi S, Gravier R. A bullet in the supraspinatus compartment successfully removed by arthroscopy: Case report and review of the literature. Case Rep Orthop 2015;2015:806735. retained foreign body, as it provides unmatched visualization, fluid irrigation throughout all available joint spaces, as well the ability to address large retained foreign bodies. Considering the location of the bullet, open retrieval may have necessitated an alternative posterior approach; however, with proper planning, even these larger retained foreign bodies were removed safely through arthroscopy. In this case, the significant glenoid loss did not result in any instability, even on a patient who primarily uses his upper extremities for weight-bearing purposes. This is mainly due to the fact it was a penetrating injury without any soft-tissue disruptions (for example, in shoulder dislocations) and the location of the humeral and glenoid bone defects.

\section{Clinical Message}

Shoulder arthroscopy can be successfully employed to retrieve larger embedded bullet or foreign bodies with the help of tools like laparoscopic graspers. Even with significant glenoid bone defect, the stability of the shoulder joint does not compromise if the bone defects are located posterosuperiorly.

6. Otero F, Cuartas E. Arthroscopic removal of bullet fragments from the subacromial space of the shoulder. Arthroscopy 2004;20:754-6.

7. Primm DD. Lead arthropathy--progressive destruction of a joint by a retained bullet. Case report. J Bone Joint Surg Am 1984;66:292-4.

8. Hartford JM, Gorczyca JT. Late arthroscopic debridement of metal fragments and synovectomy after penetrating knee joint injury by low-velocity missile: A report of two cases. J Orthop Trauma 2001;15:222-4.

9. Servien E, Walch G, Cortes ZE, Edwards TB, O'Connor DP. Posterior bone block procedure for posterior shoulder instability. Knee Surg Sports Traumatol Arthrosc 2007;15:1130-6.

10. Cerciello S, Visonà E, Morris BJ, Corona K. Bone block procedures in posterior shoulder instability. Knee Surg Sports Traumatol Arthrosc 2016;24:604-11.

Conflict of Interest: Nil Source of Support: Nil

Consent: The authors confirm that informed consent was obtained from the patient for publication of this case report
How to Cite this Article

Guevara J, Chinnakkannu K, Raju S. Acute Arthroscopic Removal of a Bullet Embedded in the Glenoid: A Case Report. Journal of Orthopaedic Case Reports 2021 March;11(3): 59-62 